\title{
Comparative Study of Esterase and Hemolytic Activities in Clinically Important Candida Species, Isolated From Oral Cavity of Diabetic and Non-diabetic Individuals
}

\author{
Mahnaz Fatahinia ${ }^{1, *}$; Farzad Poormohamadi ${ }^{1}$; Ali Zarei Mahmoudabadi ${ }^{1,2}$ \\ ${ }_{1}^{1}$ Department of Medical Mycology, School of Medicine, Ahvaz Jundishapur University of Medical Sciences, Ahvaz, IR Iran \\ ${ }^{2}$ Health Research Institute, Infectious and Tropical Diseases Research Centre, Ahvaz Jundishapur University of Medical Sciences, Ahvaz, IR Iran \\ ${ }^{*}$ Corresponding author: Mahnaz Fatahinia, Department of Medical Mycology, School of Medicine, Ahvaz Jundishapur University of Medical Sciences, Ahvaz, IR Iran. Tel: +98- \\ 6133367543, Fax:+98-6133332036, E-mail: Fatahinia@yahoo.com
}

Received: June 2, 2014; Revised: November 10, 2014; Accepted: February 16, 2015

\begin{abstract}
Background: Diabetes mellitus as a chronic metabolic disease occurs in patients with partial or complete deficiency of insulin secretion or disorder in action of insulin on tissue. The disease is known to provide conditions for overgrowth of Candida species. Candida spp. cause candidiasis by many virulence factors such as esterase, hemolysin and phospholipase.

Objectives: This study aimed to compare esterase and hemolytic activity in various Candida species isolated from oral cavity of diabetic and non-diabetic individuals.

Patients and Methods: Swab samples were taken from 95 patients with diabetes ( 35 men and 60 women) and 95 normal persons ( 42 men and 53 women) and cultured on Sabouraud dextrose agar. Identification of isolated yeasts was performed by germ tube test, morphology on CHROMagar Candida medium, corn meal agar and ability to grow at $45^{\circ} \mathrm{C}$. Hemolysin activity was evaluated using blood plate assay and esterase activity was determined using the Tween 80 opacity test.

Results: Different Candida species were isolated from 57(60\%) diabetic and 24(25\%)non-diabetic individuals. Esterase activity was detected in all Candida isolates. Only $21.6 \%$ of C. albicans from patients with diabetes had esterase activity as +3 , while it ranged from +1 to +2 in others. Hemolytic activity was determined in C. albicans, C. dubliniensis, C. glabrata and C. krusei as 0.79, 0.58, 0.66 and 0.74, respectively. Hemolytic activity was significantly different in the two groups of diabetics and non-diabetics.

Conclusions: Oral carriage of C. albicans in the diabetic group $(n=42 ; 66.7 \%)$ was significantly greater than the control group $(n=16 ; 57.1 \%)$. Esterase activity of C. albicans in diabetic group was higher than non-diabetic group. Although C. albicans remains the most frequently pathogenic yeast for human, but other species are increasing.
\end{abstract}

Keywords: Esterase; Hemolysin Factor; Candida albicans; Diabetes Mellitus

\section{Background}

Diabetes is one of the global problems, especially in developing countries. Diabetes mellitus (DM) as a chronic metabolic disease is one of the most prevalent causes of mortality in low-income and middle-income countries (1). Patients with diabetes are more sensitive to certain infections. Today, it has been proven that oral cavity can act as a primary site for dissemination of microorganisms including Candida species (2). Oral candidiasis is one of the most common opportunistic infections in patients with diabetes and immunodeficiency (3). According to a report by Mohammadi, et al. in Iran, from 855 yeast strains isolated from different clinical specimens, Candida albicans was the predominant species (58.6\%) followed by C. parapsilosis (11.0\%), C. glabrata (8.3\%), C. tropicalis (7.0\%), C. kefyr (5.8\%), C. krusei (4.4\%), C. orthopsilosis (2.1\%) and C. guilliermondii (0.6\%). A few strains of C. lusitaniae, C. rugosa, C. intermedia, C. inconspicua, Cryptococcus neoformans and Saccharomyces cerevisiae were isolated (4).
Although C. albicans is the most common cause of candidiasis, but 12 to 14 species of non-albicans Candida are known to cause such infections. Recent epidemiological studies have shown that non -C. albicans Candida species (NACs) such as C. tropicalis, C. parapsilosis, C. krusei and C. glabrata have repeatedly emerged as human pathogens (4). Candida species, especially C. albicans tend to colonize oral cavities, secrete hydrolytic enzymes and cause inflammation and hyperplasia of the gums and mouth, which can lead to discomfort, pain and disseminated infection in patients with impaired immune system. Candida species secrete different lytic enzymes such as phospholipases, esterases and hemolysin factor which digest surface membrane of the host cells, facilitate binding and penetration of microorganisms into the host tissue and contribute to tissue invasion, thus play important roles in Candida pathogenicity (5). They also attack host immune cells and molecules and prevent their antimicrobial activity.

Copyright (C) 2015, Ahvaz Jundishapur University of Medical Sciences. This is an open-access article distributed under the terms of the Creative Commons Attribution-NonCommercial 4.0 International License (http://creativecommons.org/licenses/by-nc/4.0/) which permits copy and redistribute the material just in noncommercial usages, provided the original work is properly cited. 
Hemolysin of Candida species is amidst the most frequently studied enzymes produced by fungi, which is released during iron acquisition and facilitates the hyphal invasion in disseminated candidiasis (6). To our knowledge, secretion of hemolysin by Candida isolates from patients with diabetes had not been studied before. Detection of secretory enzymes in Candida strains isolated from DM can be an important step for management of these patients. Additionally, most previous researches focused on C. albicans.

\section{Objectives}

We aimed to:

1) Isolate, identify and compare enzymatic activities in Candida species isolated from diabetic and non- diabetic patients referred to Imam Sajjad Diabetes Center

2) Evaluate enzymatic activity in both C. albicans and NACs, especially C. glabrata, C. tropicalis and C. krusei.

\section{Patients and Methods}

\subsection{Patients}

Ninety-five patients with diabetes mellitus (35 men and 60 women) and 95 healthy volunteers (42 men and 53 women) were included in the study and matched for age, sex, dental status and smoking habits. The study protocol was approved by the research ethics committee (Ahvaz Jundishapur University of Medical Sciences). Selected patients from local outpatient diabetes clinics aged 10 to 85 years. After getting informed consent, patients referred to Imam Sajjad Diabetes Center (Dehdasht-Iran, 2013) were sampled using sterile swabs.

\subsection{Candida Isolates}

Swab samples were dispensed onto CHROMagar media (CHROMagar Candida, Paris, France) and incubated at $37^{\circ} \mathrm{C}$ for 48 hours. Candida species were determined by colony color on CHROMAgar medium, germ tube test, growth at $45^{\circ} \mathrm{C}$ and microscopic morphology after growing on corn meal agar medium.

\subsection{Esterase Assay}

Esterase activity was measured using the Tween 80 opacity test medium, which was prepared with $10 \mathrm{~g}$ of bacteriological peptone (Merck, Germany), $5 \mathrm{~g}$ of sodium chloride, $0.1 \mathrm{~g}$ of calcium chloride, $15 \mathrm{~g}$ of agar and 1000 $\mathrm{mL}$ of distilled water. After, the medium was autoclaved; it was cooled to about $50^{\circ} \mathrm{C}$ and was added $5 \mathrm{~mL}$ of autoclaved Tween 80 (Merck, Germany). The inoculated agar plates were incubated at $30^{\circ} \mathrm{C}$ and examined daily for 10 days. All inoculations were performed in duplicate. The colony diameter (a) and the diameter of colony plus precipitation zone (b) were measured. The esterase activities were expressed as Ez Value (a/b) as described by Price et al. (7).

\subsection{Hemolysin Assay}

Hemolysin production was evaluated using a modification of the plate assay described by Manns et al. (8). In brief, the isolates cultured on Sabouraud glucose agar were spot inoculated on a sugar-enriched sheep blood agar medium to yield a circular inoculation site of about $5 \mathrm{~mm}$ in diameter. The latter medium was prepared by adding 5 - $7 \mathrm{~mL}$ of fresh sheep blood (Darvash Co. Iran) to $100 \mathrm{~mL}$ of Sabouraud glucose agar supplemented with 3\% glucose (Merck, Germany). The plates were incubated at $37^{\circ} \mathrm{C}$ in $5 \% \mathrm{CO}_{2}$ for 48 hours. The ratio of the diameter of the colony to that of the translucent zone of hemolysis (in $\mathrm{mm}$ ) was used as the hemolytic index (Hz Value) (9).

\section{Results}

Candida spp. were isolated from 57 patients with diabetes (60\%) and 24 non-diabetic persons (25.5\%). The oral carriage of C. albicans in the diabetic group $(n=42 ; 66.7 \%)$ was significantly greater than that in the control group ( $\mathrm{n}=16 ; 57.1 \%$ ) (Chi-square test, $\mathrm{P}=0.05)$. Other Candida species isolated included C. glabrata (9 isolates from DM and four from control groups), C. dubliniensis (eight isolates from DM and four from control groups), C. krusei (four isolate from DM and the control group) (Table 1).

Table 1. Prevalence of Candida Species in Diabetic and NonDiabetic Groups ${ }^{\text {a }}$

\begin{tabular}{|c|c|c|c|c|}
\hline Variable & C. albicans & C. dubliniensis & C. krusei & C. glabrata \\
\hline Diabetic & $42(66.7)$ & $8(12.7)$ & $9(14.3)$ & $4(6.4)$ \\
\hline Non-Diabetic & $16(57.1)$ & $4(14.3)$ & $4(14.3)$ & $4(14.3)$ \\
\hline
\end{tabular}

${ }^{\mathrm{a}}$ Data are presented No.(\%)

Hemolytic activity was detected in $100 \%$ and $52 \%$ of the isolates from diabetic and non-diabetic groups. Although $75 \%$ of C. albicans isolates from non-diabetic group produced hemolysin, the total hemolysin activity of Candida species was significantly higher in diabetic group than non-diabetic group (Table 2).

Table 2. Hemolytic Activity (Hz Value) of Oral Candida Species Isolates From Diabetic and Non-Diabetic Subjects

\begin{tabular}{lccccc}
\hline Variable & C. albicans & C. dubliniensis & C. krusei & C. glabrata & Total \\
\hline Diabetic & 0.72 & 0.59 & 0.65 & 0.72 & 0.67 \\
$\begin{array}{l}\text { Non-Dia- } \\
\text { betic }\end{array}$ & 0.78 & 0.93 & 1 & 0.94 & 0.91 \\
\hline
\end{tabular}

After grading esterase activity in various Candida species, it was found that only C. glabrata and C. albicans were able to demonstrate esterase activity at +3 level. In nondiabetic, $50 \%$ of C. glabrata and C. krusei produced esterase at +1 level, although all of C. dubliniensis and $87.5 \%$ of C. albicans produced it at +2 level (Figure 1 and Table 3 ). 


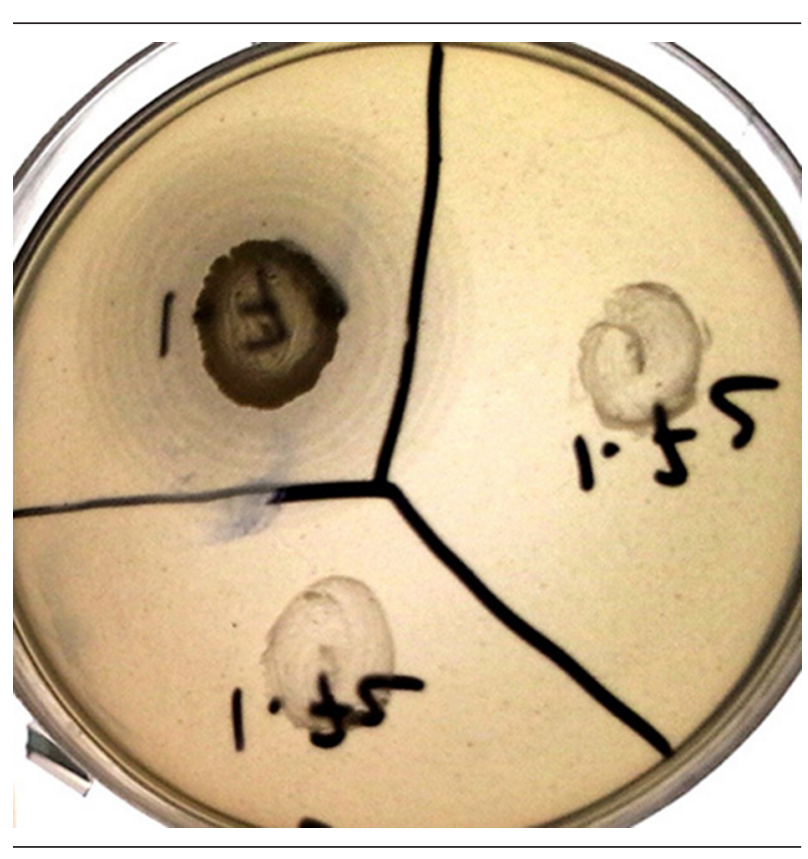

Figure 1. Esterase Activity of Candida Species

Table 3. Esterase Activity (Ez Value) of Oral Candida Species Isolates From Diabetic and Non-Diabetic Subjects ${ }^{a}$, b

\begin{tabular}{|ccc}
\hline Variables & Diabetic & Non-Diabetic \\
\hline C. krusei & - & 50 \\
1 & 100 & 50 \\
2 & - & - \\
3 & - & \\
C. glabrata & 66.7 & 50 \\
1 & 33.3 & 50 \\
\hline 2 & & - \\
3 & 25 & - \\
\hline C. dubliniensis & 75 & 100 \\
\hline 1 & - & - \\
\hline 2 & & \\
\hline 3 & 8.1 & 12.5 \\
\hline C. albicans & 70.3 & 87.5 \\
\hline 1 & 21.6 & - \\
\hline 2 & & \\
\hline 3 & & \\
\hline
\end{tabular}

a Score of esterase: $+1=0.7-0.99 ;+2=0.4-0.69 ;+3=0.1-0.39$

$\mathrm{b}$ Data are presented as \%.

\section{Discussion}

The purpose of this research was to identify and assess various species of Candida in oral cavity of diabetic and non-diabetic individuals and to compare their virulence factors such as esterase and hemolysin enzymes with the assumption that virulence factors of Candida species iso- lated from diabetic patients are higher than the control group. In this research, Candida was isolated from $60 \%$ of diabetic patients versus $25.5 \%$ of normal subjects. In patients with DM, due to reduced salivary flow, increased blood glucose concentration and decreased saliva $\mathrm{pH}$ the possibility of adherence and colonization of Candida is increased in mouth. The results of this study confirmed previous studies $(2,10,11)$.

The most frequent oral yeast isolates from the study subjects was C. albicans. $66.7 \%$ of diabetic patients and $57.1 \%$ of normal subjects had C. albicans. Based on previous studies, the most common pathogen obtained in adults with oral mucosal was C. albicans (11). The oral carriage of C. albicans in the diabetic group ( $n=42 ; 66.7 \%$ ) was significantly greater than the control group ( $\mathrm{n}=16 ; 57.1 \%$ ) (Chi-square test, P, 0.05). Manfredi et al. reported that Candida grew out from the oral of diabetic subjects and non-diabetics by $60.5 \%$ and $57.3 \%$, respectively (11). The highest growth rates related to C. albicans from the oral cavity of diabetic subjects (83.3\%) and normal group (76.9\%).

In our study, the percentage of C. albicans isolates from both groups was lower than report of Manfredi et al. (11). These results may be due to lower average age of our subjects, living conditions and some host dietary habits. However, Soysa et al. in a study on diabetic patients reported isolation of C. albicans between 18 - 80\%, which is in accordance with our results (12). In this research, $C$. dubliniensis was isolated from eight of 95 patients with diabetes (8.4\%). Willis et al. in a study isolated $18.2 \%$ C. dubliniensis from insulin-dependent diabetics (13), while Manfredi (11), showed it 3.5\%. Previously, it was thought that this species of Candida is only separated from patients with HIV.

Four C. glabrata isolates were isolated from each of the two groups of patients and controls. Isolation of these species was higher in healthy subjects than patients with diabetes (Table 1). Studies on hemolysin activity of Candida species isolated from patients with diabetes are limited. Hemolysin activity in our study was significantly higher in the diabetic group than the control group. Hemolytic activity was assayed in C. albicans, C. dubliniensis, C. glabrata and C. krusei as 0.72, 0.59, 0.65 and 0.79, respectively. Hemolytic activity was detected in $100 \%$ of the Candida species in diabetic group, while only $52 \%$ of Candida isolates in non-diabetic group showed hemolytic activity. Of course, $75 \%$ of C. albicans isolated from non-diabetic group produced hemolysin factor. The hemolytic activity of $C$. albicans was significantly higher than that of non- $C$. albicans Candida isolates $(\mathrm{P}=0.0001)$ in control group, but was not in the diabetic group (Table 2).

Our findings indicated that non-C. albicans isolates in patients with diabetes may possess greater virulence potential than non-albicans species in the control group. Manns et al. found that C. albicans does not show any hemolytic activity when there is no glucose available in the medium. They stated that adding glucose to the culture medium increases the hemolytic activity. Therefore, it 
can be predicted that increasing glucose concentrations in saliva would enhance hemolysin production, adhesion and colonization of Candida species in oral cavity of diabetic subjects (8). In this study, each of the Candida species in diabetic group significantly showed higher hemolytic activity the same as normal subjects. The differences in the hemolytic activities of non-albicans species were further. Perhaps these species in abnormal conditions such as diabetes had more pathogenicity. Shimizu et al. injected them into mice and examined the virulence of different strains and confirmed that production of extracellular enzymes by Candida isolated from patients with diabetes is higher than normal individuals (14). Luo et al. examined the hemolytic activity of 14 species of Candida separately. They showed the ability of Candida species in production of one or both hemolysins, but there was no quantitative report regarding the hemolytic activity of Candida species in patients with diabetes (15) and our research was the first in this regard. Aktas et al. evaluated esterase activity in different species of Candida qualitatively. In this study, almost all species produced sediment in Tween 80 opacity test medium after 2 - 10 days (16). This test is confirmed by other researchers, because it is convenient, economical and easy to perform (17). Kumar et al. qualitatively surveyed the esterase activity of Candida species isolated from immunocompromised hosts (18). Slifkim believed that due to lack of esterase production on Tween 80 opacity test medium by C. dubliniensis could identify C. dubliniensis from C. albicans, whereas in our study $100 \%$ of C. dubliniensis produced esterase (17). In our study, esterase activity was quantitatively evaluated. $21.6 \%$ of C. albicans strains and $33.3 \%$ of C. glabrata strains isolated from patients with diabetes produced esterase at level +3 , whereas these two species in the control group showed esterase activity at levels +1 and +2 (Table 3 ).

It is concluded that esterase and hemolytic activities of Candida in patients with diabetes are higher than healthy subjects, but some species such as C. glabrata act similar to C. albicans in diabetes.

\section{Acknowledgements}

We appreciate Imam Sajjad Diabetes Center staff who helped us to prepare patient samples.

\section{Authors' Contributions}

1- Mahnaz Fatahinia (Corresponding Author): Study concept and design, analysis and interpretation of data, drafting of the manuscript, administrative, technical and material supports, study supervision and statistical analysis. 2- Farzad Poormohamadi: Acquisition of data and technical support. 3- Ali Zarei Mahmoudabadi: Criti- cal revision of the manuscript for important intellectual content and material support.

\section{Financial Disclosure}

This study was a student research. All costs allocated to it by the Research Council were spent to prepare materials and equipment needed for the tests.

\section{Funding/Support}

This study was part of student researches (92/3/22 No: 92s.8) supported by Deputy of Research Ahvaz Jundishapur University of Medical Sciences.

\section{References}

1. $\quad$ Diabetes-a global threat. Lancet. 2009;373(9677):1735.

2. Azizi A, Rafiei A, Fakhredini A, Lvaf S. . Comparison of oral strains of Candida and $\mathrm{PH}$ of saliva in diabetics and non-diabetics patients. Jundishapur Sci Med J. 2010;6(54):1-2.

3. Li X, Kolltveit KM, Tronstad L, Olsen I. Systemic diseases caused by oral infection. Clin Microbiol Rev. 2000;13(4):547-58.

4. Mohammadi R, Mirhendi H, Rezaei-Matehkolaei A, Ghahri M Shidfar MR, Jalalizand N, et al. Molecular identification and distribution profile of Candida species isolated from Iranian patients. Med Mycol. 2013;51(6):657-63.

5. Schaller M, Borelli C, Korting HC, Hube B. Hydrolytic enzymes as virulence factors of Candida albicans. Mycoses. 2005;48(6):365-77.

6. Weinberg ED. Iron and infection. Microbiol Rev.1978;42(1):45-66.

7. Price MF, Wilkinson ID, Gentry LO. Plate method for detection of phospholipase activity in Candida albicans. Sabouraudia. 1982;20(1):7-14.

8. Manns JM, Mosser DM, Buckley HR. Production of a hemolytic factor by Candida albicans. Infect Immun.1994;62(11):5154-6.

9. Tsang CS, Chu FC, Leung WK, Jin LJ, Samaranayake LP, Siu SC. Phospholipase, proteinase and haemolytic activities of Candida albicans isolated from oral cavities of patients with type 2 diabetes mellitus. J Med Microbiol. 2007;56(Pt 10):1393-8.

10. Belazi M, Velegraki A, Fleva A, Gidarakou I, Papanaum L, Baka D, et al. Candidal overgrowth in diabetic patients: potential predisposing factors. Mycoses. 2005;48(3):192-6.

11. Manfredi M, McCullough MJ, Al-Karaawi ZM, Hurel SJ, Porter SR. The isolation, identification and molecular analysis of Candida spp. isolated from the oral cavities of patients with diabetes mellitus. Oral Microbiol Immunol. 2002;17(3):181-5.

12. Soysa NS, Samaranayake LP, Ellepola AN. Diabetes mellitus as a contributory factor in oral candidosis. Diabet Med. 2006;23(5):455-9.

13. Willis AM, Coulter WA, Sullivan DJ, Coleman DC, Hayes JR, Bell PM, et al. Isolation of C. dubliniensis from insulin-using diabetes mellitus patients.J Oral Pathol Med. 2000;29(2):86-90.

14. Shimizu MT, Almeida NQ, Fantinato V, Unterkircher CS. Studies on hyaluronidase, chondroitin sulphatase, proteinase and phospholipase secreted by Candida species. Mycoses. 1996;39(5-6):161-7.

15. Luo G, Samaranayake LP, Yau JY. Candida species exhibit differen tial in vitro hemolytic activities. J Clin Microbiol. 2001;39(8):2971-4.

16. Aktas E, Yigit N, Ayyildiz A. Esterase activity in various Candida species. JInt Med Res. 2002;30(3):322-4.

17. Slifkin M. Tween 80 opacity test responses of various Candida species. JClin Microbiol. 2000;38(12):4626-8.

18. Kumar CP, Menon T, Sundararajan T, Nalini S, Thirunarayan MA, Rajasekaran S, et al. Esterase activity of Candida species isolated from immunocompromised hosts. Rev Iberoam Micol. 2006;23(2):101-3. 